\title{
Structural basis of the interspecies interaction between the chaperone DnaK(Hsp70) and the co-chaperone GrpE of archaea and bacteria ${ }^{\star}$
}

\author{
Michał A. Żmijewski ${ }^{1}$, Joanna Skórko-Glonek ${ }^{1}$, Fabio Tanfani ${ }^{2}$, Bogdan Banecki ${ }^{3}$, \\ Agnieszka Kotlarz ${ }^{1}$, Alberto J.L. Macario ${ }^{4 凶}$ and Barbara Lipińska ${ }^{1 凶}$ \\ ${ }^{1}$ Department of Biochemistry, University of Gdansk, Gdańsk, Poland; ${ }^{2}$ Institute of Biochemistry, Faculty of \\ Sciences, Università Politecnica delle Marche, Ancona, Italy; ${ }^{3}$ Department of Molecular and Cellular Biology, \\ Faculty of Biotechnology, University of Gdansk, Gdansk, Poland; ${ }^{4}$ Center of Marine Biotechnology, University \\ of Maryland Biotechnology Institute, Baltimore, Maryland, USA
}

Received: 18 March, 2007; revised: 14 May, 2007; accepted: 08 June, 2007 available on-line: 12 June, 2007

\begin{abstract}
Hsp70s are chaperone proteins that are conserved in evolution and present in all prokaryotic and eukaryotic organisms. In the archaea, which form a distinct kingdom, the Hsp70 chaperones have been found in some species only, including Methanosarcina mazei. Both the bacterial and archaeal Hsp70(DnaK) chaperones cooperate with a GrpE co-chaperone which stimulates the ATPase activity of the DnaK protein. It is currently believed that the archaeal Hsp70 system was obtained by the lateral transfer of chaperone genes from bacteria. Our previous finding that the DnaK and GrpE proteins of $M$. mazei can functionally cooperate with the Escherichia coli GrpE and DnaK supported this hypothesis. However, the cooperation was surprising, considering the very low identity of the GrpE proteins (26\%) and the relatively low identity of the DnaK proteins (56\%). The aim of this work was to investigate the molecular basis of the observed interspecies chaperone interaction. Infrared resolution-enhanced spectra of the $M$. mazei and E. coli DnaK proteins were almost identical, indicating high similarity of their secondary structures, however, some small differences in band position and in the intensity of amide $I^{\prime}$ band components were observed and discussed. Profiles of thermal denaturation of both proteins were similar, although they indicated a higher thermostability of the $M$. mazei DnaK compared to the E. coli DnaK. Electrophoresis under non-denaturing conditions demonstrated that purified DnaK and GrpE of E. coli and M. mazei formed mixed complexes. Protein modeling revealed high similarity of the 3-dimensional structures of the archaeal and bacterial DnaK and GrpE proteins.
\end{abstract}

Keywords: archaeal DnaK structure, archaeal Hsp70(DnaK), ATPase domain, DnaK-GrpE complex, molecular chaperones, substrate-binding domain

\section{INTRODUCTION}

The Hsp70 protein is a molecular chaperone whose main functions are to assist in the folding of nascent polypeptides and in the re-folding of unfolded proteins, and to participate in other events related to maturation, translocation, and functioning of proteins (Bukau et al., 2000; 2006; Truscott et al.,

\footnotetext{
${ }^{\square}$ Corresponding authors: B. Lipińska, Department of Biochemistry, University of Gdansk, Kładki 24, 80-822 Gdańsk, Poland; tel.: (48 58) 305 9278; fax: (48 58) 301 5741; e-mail: lipinska@biotech.ug.gda.pl; Alberto J. L. Macario, Center of Marine Biotechnology, University of Maryland Biotechnology Institute, 701 E. Pratt Street, Baltimore, Maryland 21202, USA; tel.: (410) 234 8849; fax: (410) 234 8896; e-mail: macario@umbi.umd.edu

'Present address: Department of Pathology and Laboratory Medicine, University of Tennessee Health Science Center, 195 Manassas Ave., Memphis, Tennessee 38163, USA.

^ Preliminary results concerning current work were presented at the XXXIX Congress of the Polish Biochemical Society, Gdańsk, Poland, September 16-20, 2003.

Abbreviations: DnaK $E_{E c^{\prime}}$ DnaK protein of E. coli; DnaK ${ }_{M m^{\prime}}$ DnaK protein of M. mazei; FT-IR, Fourier transform infrared; $\operatorname{GrpE}_{E \mathcal{C}^{\prime}}$ GrpE protein of E. coli; $\mathrm{GrpE}_{M m^{\prime}}$ GrpE protein of M. mazei; SBD, substrate-binding domain of DnaK protein.
} 
2003; Deuerling \& Bukau, 2004; Young et al., 2004; Kultz, 2005). The Hsp 70 proteins are well conserved in evolution and have been found both in prokaryotic and eukaryotic organisms. In prokaryotes, Hsp70, customarily termed DnaK, interacts with Hsp40 (DnaJ) and with the nucleotide-exchange factor GrpE to carry out the chaperoning activities which require energy from ATP.

The DnaK molecule has specialized domains for recognizing and binding substrates (polypeptides), binding and hydrolyzing ATP, and interacting with DnaJ and GrpE. These domains have been identified and their properties have been studied in several DnaKs, particularly that from Escherichia coli. DnaK consists of an about $44 \mathrm{kDa}$ amino-terminal ATPase domain and an about $27 \mathrm{kDa}$ carboxy-terminal substrate binding domain (SBD). The three-dimensional architecture of the ATPase domain (Harrison et al., 1997) and that of the SBD (Zhu et al., 1996) have been determined. The ATPase domain is composed of two lobes that form a cleft for ATP binding. In addition, this domain binds the nucleotide-exchange factor GrpE. The SBD consists of two separated regions: the $\beta$-sandwich subdomain with a cavity which accommodates the polypeptide, and the $\alpha$-helical subdomain, forming a latch segment or lid, closing on top of the substrate-binding cavity (Zhu et al., 1996).

DnaK has a weak ATPase activity and it cycles between ATP- and ADP-bound stages, with its affinity for the polypeptide substrate being lower in the former than in the ADP-bound stage. The cycling of DnaK between these stages is regulated by the co-chaperone DnaJ and the nucleotide-exchange factor GrpE (the latter functions as a homodimer). The DnaJ protein binds to DnaK and accelerates hydrolysis of ATP by DnaK, thus facilitating the binding of the substrate polypeptide. GrpE induces release of ADP from DnaK and, upon rebinding of ATP, the DnaK-polypeptide complex dissociates and the folded protein is released. This completes the reaction cycle and leaves the substrate-binding cavity free and open to receive another polypeptide (Bukau et al., 2000; 2006; Mayer et al., 2000; Deuerling \& Bukau, 2004; Erbse et al., 2004; Young et al., 2004).

Archaeal organisms form an independent kingdom and possess a mixture of eukaryotic and prokaryotic features. They frequently inhabit environments with extremely high temperatures or very high saline content. Surprisingly, the Hsp70 system is not present in all the archaeal species - it has been found mainly in those which live at moderate temperatures, including the methanogenic archaeon Methanosarcina mazei. It is currently believed that the archaea which possess the DnaK-DnaJ-GrpE chaperoning system gained it by the lateral transfer of the genes from bacteria (Gribaldo et al., 1999; Ma- cario \& Conway De Macario, 1999; 2001; Macario et al., 2004). This theory is based on the DNA sequence analysis and recently a functional similarity of the M. mazei and E. coli DnaK systems has been shown, which supports the theory. We have found that M. mazei DnaK $\left(\operatorname{DnaK}_{M m}\right)$ and GrpE $\left(\mathrm{GrpE}_{M m}\right)$ are able to function efficiently with the $E$. coli cochaperone GrpE $\left(\mathrm{GrpE}_{E c}\right)$ and $\operatorname{DnaK}\left(\operatorname{DnaK}_{E c}\right)$ in the reactivation of thermally denatured luciferase, and that $\mathrm{GrpE}_{M m}$ can replace $\mathrm{GrpE}_{E c}$ in vivo in the heat shock response and in promotion of bacteriophage $\lambda$ growth (Zmijewski et al., 2004). The demonstration of these functional in vivo and in vitro interactions indicated that $\mathrm{DnaK}_{M m}$ was able to physically interact with $\mathrm{GrpE}_{E c}$ and, vice versa, DnaK $E c$ could also interact with $\mathrm{GrpE}_{\mathrm{Mm}}$. This was rather surprising, considering the very low identity of the GrpE proteins $(26 \%)$ and the relatively low identity of the DnaK proteins (56\%).

The aim of this work was to further investigate the molecular basis of the interspecies cooperation of the DnaK and GrpE proteins of E. coli and M. mazei. Using Fourier-transform infrared (FT-IR) spectroscopy we were able to show a high similarity of the secondary structures of the bacterial and archaeal DnaKs. Formation of the interspecies DnaK-GrpE complexes was demonstrated by native electrophoresis. Molecular modeling of the $M$. mazei DnaK domains and of GrpE, basing on the solved structures of their E. coli counterparts, was performed to better understand the experimentally shown similarities and interactions.

\section{MATERIALS AND METHODS}

Chemicals. Deuterium oxide $\left(99.9 \%{ }^{2} \mathrm{H}_{2} \mathrm{O}\right)$, ${ }^{2} \mathrm{HCl}$, and $\mathrm{NaO}^{2} \mathrm{H}$ were purchased from Aldrich (Sigma-Aldrich S.r.l., Milan, Italy). All other chemicals were commercial products of the purest quality purchased from Sigma (Poznań, Poland), or were obtained as indicated in the text.

Proteins and native electrophoresis. DnaK and GrpE proteins from M. mazei and E. coli were purified as described previously (Zmijewski et al., 2004). All proteins were dialyzed against $25 \mathrm{mM}$ Hepes, $100 \mathrm{mM} \mathrm{KCl}, 10 \%$ glycerol, pH 7.2. Protein ( $>95 \%$ pure) concentrations were determined by the Bradford method (Bradford, 1976) and were confirmed by densitometry of Coomassie-stained sodium dodecyl sulphate polyacrylamide-gel electrophoresis (SDS/PAGE) gels. DnaK preparations were free of ATP, as tested by the malachite green method as we described before (Zmijewski et al., 2004). Native gel electrophoresis was performed using the Laemmli system (Laemmli, 1970) without SDS and without a stacking gel, in 10\% resolving gels. Before the native 
electrophoresis, DnaK $\mathrm{K}_{M m^{\prime}} \mathrm{DnaK}_{E c^{\prime}} \mathrm{GrpE}_{M m}$ or $\mathrm{GrpE}_{E c}$ were incubated for $30 \mathrm{~min}$ in $50 \mathrm{mM}$ Hepes, $\mathrm{pH} 7.5$, $50 \mathrm{mM} \mathrm{KCl}$, and $10 \mathrm{mM} \mathrm{MgCl}{ }_{2}$ buffer in different combinations as indicated in figure legends.

Protein modeling. Modeling of M. mazei chaperone proteins was done by the Swiss Model server and Deep View/Swiss-PdbViewer (Peitsch, 1996; Guex \& Peitsch, 1997). Models of the domains of the DnaK ${ }_{M m}$ protein were done with E. coli structures used as templates (1DKGD for the ATPase domain, and 1DKXA for the SBD). The model of the $\mathrm{GrpE}_{M m}$ dimer was based on the structure of the E. coli GrpE complex with the ATPase domain of $D{ }^{\prime} K_{E c^{\prime}}$ and each molecule of the dimer was folded separately (1DKGA, 1DKGB). After modeling, the structures of the $M$. mazei proteins were minimized with the GROMOS96 force field implementation of Swiss-PdbViewer.

Preparation of samples for infrared spectroscopy. Typically, $1.5 \mathrm{mg}$ of protein, dissolved in the buffer used for its purification, was centrifuged in a "30 K Centricon" micro concentrator (Millipore) at $5000 \times g$ at $4^{\circ} \mathrm{C}$ and was concentrated into a volume of $40 \mu \mathrm{l}$. Then, $300 \mu \mathrm{l}$ of $25 \mathrm{mM}$ Hepes, $50 \mathrm{mM} \mathrm{NaCl}$, $3 \mathrm{mM}$ dithiothreitol buffer, prepared in ${ }^{2} \mathrm{H}_{2} \mathrm{O}$ p ${ }^{2} \mathrm{H}$ 7.2, was added and the sample was concentrated again. The $\mathrm{p}^{2} \mathrm{H}$ value corresponds to the $\mathrm{pH}$ meter reading +0.4 (Salooma et al., 1964). The concentration-and-dilution procedure was repeated several times in order to completely replace the original buffer with the Hepes buffer. In the last washing, the protein solution was concentrated by decreasing its volume down to $35 \mu \mathrm{l}$ and was used for FT-IR analysis.

Fourier-transform infrared spectroscopy. The concentrated protein samples were placed in a thermostated Graseby Specac 20500 cell (Graseby-Specac Ltd, Orpington, Kent, UK) fitted with $\mathrm{CaF}_{2}$ windows and a $25-\mu \mathrm{m}$ Teflon spacer. FT-IR spectra were recorded by means of a Perkin-Elmer 1760-x Fourier transform infrared spectrometer using a deuterated triglycine sulphate detector and a normal Beer-Norton apodization function. For at least $24 \mathrm{~h}$ before and during data acquisition, the spectrometer was continuously purged with dry air at a dew point of $-40^{\circ} \mathrm{C}$. Spectra of buffers and samples were acquired at $2 \mathrm{~cm}^{-1}$ resolution under the same scanning and temperature conditions. In the thermal denaturation experiments, the temperature was raised in $5^{\circ} \mathrm{C}$ steps from $20^{\circ} \mathrm{C}$ to $95^{\circ} \mathrm{C}$. The cell was maintained at the desired temperature using an external bath circulator (HAAKE F3), and the actual temperature in the cell was controlled by a thermocouple placed directly onto the window. Spectra were collected and processed using the "Spectrum" software from Perkin Elmer.

Correct subtraction of ${ }^{2} \mathrm{H}_{2} \mathrm{O}$ was adjusted to the removal of the ${ }^{2} \mathrm{H}_{2} \mathrm{O}$ bending absorption close to $1220 \mathrm{~cm}^{-1}$ (Tanfani et al., 1997). The deconvoluted parameters for the amide I' band were set with a gamma value of 2.5 and a smoothing length of 50 . Second-derivative spectra were calculated over a 9data-point range $\left(9 \mathrm{~cm}^{-1}\right)$. The midpoint transition in thermal denaturation was calculated as described (Meersman et al., 2002).

\section{RESULTS}

\section{Secondary structure and thermal stability of DnaK proteins}

We expected that the interspecies interaction of the DnaK and GrpE proteins should be based on a three-dimensional similarity of the archaeal and bacterial proteins. In order to learn more about the overall secondary structures of the DnaK chaperones, we applied Fourier-transform infrared (FT-IR) spectroscopy.

The amide I' band of the spectrum of a protein is broad $\left(1700-1620 \mathrm{~cm}^{-1}\right)$, and it is composed of various bands due to the absorption of different secondary-structural elements (Byler \& Susi, 1986; Arrondo et al., 1993). The amide I' component bands can be revealed through application of resolutionenhancement methods (deconvolution and/or second derivation) to the original absorbance spectrum (Byler \& Susi, 1986; Arrondo et al., 1993). Figure 1A displays the second derivative and deconvolved infrared spectra of $\mathrm{DnaK}_{M m}$ and $\mathrm{DnaK}_{E c}$ in the 1750$1500 \mathrm{~cm}^{-1}$ range. In the $1700-1620 \mathrm{~cm}^{-1}$ interval, the spectra show the same amide I' component bands, which indicates very high similarity of the secondary structures of the DnaKs of M. mazei and E. coli. However, there are some minor differences in the position and intensity of the bands, suggesting small differences in the secondary-structural composition of the two proteins. The 1638.6 (in $\mathrm{DnaK}_{E c}$ ) and $1636.0 \mathrm{~cm}^{-1}$ (in $\mathrm{DnaK}_{\mathrm{Mm}}$ ) bands are due to $\beta$-sheets, whilst the 1650.9 (in $\mathrm{DnaK}_{E c}$ ) and $1652.8 \mathrm{~cm}^{-1}$ (in $\mathrm{DnaK}_{\mathrm{Mm}}$ ) bands are due to $\alpha$-helices (Arrondo et al., 1993). The position of the $\beta$-sheet band in the $D_{n a K}$ spectrum, lower than in the DnaK $E_{E c}$ spectrum, suggests that $\beta$-sheets, or portions of them, are more exposed to the solvent $\left({ }^{2} \mathrm{H}_{2} \mathrm{O}\right.$ ) (Pedone et al., 2003) in $\mathrm{DnaK}_{M m}$ than in $\mathrm{DnaK}_{E c}$. The position of the $\alpha$-helix band in the DnaK $E_{E c}$ spectrum, lower than in the DnaK ${ }_{M m}$ spectrum, suggests that $\alpha$-helices, or portions of them, are more exposed to the solvent in DnaK $_{E c}$ than in DnaK $\mathrm{Mm}_{\mathrm{m}}$. The $1670.1 \mathrm{~cm}^{-1}$ band and the bands close to 1688 and $1680 \mathrm{~cm}^{-1}$ may be due to $\beta$-sheets and/or turns (Krimm \& Bandekar, 1986; Arrondo et al., 1993). The $1628 \mathrm{~cm}^{-1}$ shoulder, which is absent in the DnaK ${ }_{M m}$ spectrum, could be due to protein intermolecular interactions, to an unusually strongly hydrogen-bonded $\beta$-sheet, or to $\beta$-struc- 

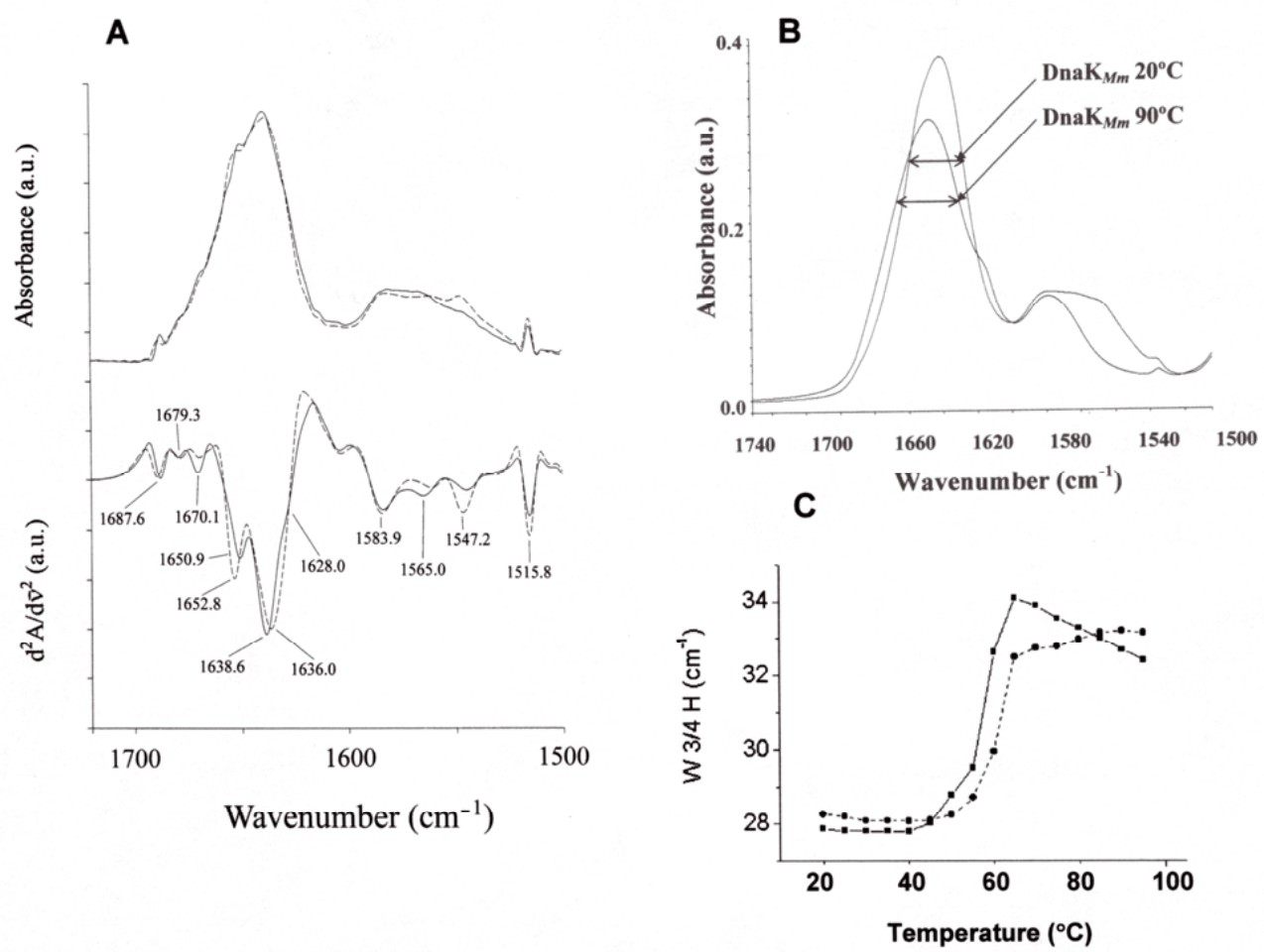

Figure 1. Comparative analyses of DnaK ${ }_{M m}$ and DnaK ${ }_{E c}$ secondary structures - FT-IR spectroscopy data.

A. Resolution-enhanced spectra of $\mathrm{DnaK}_{M m}$ and $\mathrm{DnaK}_{E c}$ over the range of infrared wavenumbers shown on the horizontal axis. Deconvolved (top graph) and second-derivative (bottom graph) spectra of DnaK $E_{E c}$ (continuous line) and DnaK${ }_{M m}$ (dashed line) at $20^{\circ} \mathrm{C}$ and $\mathrm{p}^{2} \mathrm{H}$ 7.2. B. Temperature-dependent changes of $\mathrm{DnaK}_{M m}$ infrared spectrum. The absorbance spectra of $\mathrm{DnaK}_{M m}$ at 20 and $90^{\circ} \mathrm{C}$ are shown. Amide I' bandwidths (measured at $3 / 4$ of height) are indicated. C. Thermal denaturation curves of $\mathrm{DnaK}_{M m}$ and $\mathrm{DnaK}_{E c}$ (obtained by measurment of the amide I' bandwidths at $3 / 4$ of height). Circles and squares, DnaK ${ }_{M m}$ and DnaK $E_{c^{\prime}}$ respectively. The $t_{\mathrm{m}}$ was calculated from the curves as described (Meersman et al., 2002). The $t_{\mathrm{m}}$ for $\mathrm{DnaK}_{M m}$ is 61.0 and that for $\mathrm{DnaK}_{E c}$ is $56.4^{\circ} \mathrm{C}$. All determinations and the calculation of arbitrary units (a.u.) were done as described in Materials and Methods.

tures interacting strongly with the solvent (particularly solvent-exposed $\beta$-strands), in DnaK $\mathrm{K}_{E c}$ (Jackson \& Mantsch, 1992; Arrondo et al., 1993). Hence, the lack of the $1628 \mathrm{~cm}^{-1}$ band in the DnaK $\mathrm{Mm}_{\mathrm{m}}$ spectrum indicates that the above-mentioned phenomena are absent or very minor in this protein.

The peak at $1547.2 \mathrm{~cm}^{-1}$ represents the residual amide II band (encompassing the $1600-1500 \mathrm{~cm}^{-1}$ interval) absorption, i.e., the absorption of the amide II band after ${ }^{1} \mathrm{H} /{ }^{2} \mathrm{H}$ exchange of the amide hydrogens of the polypeptide chain. The higher intensity of the residual amide II band in the $\mathrm{DnaK}_{M m}$ spectrum indicates that the ${ }^{1} \mathrm{H} /{ }^{2} \mathrm{H}$ exchange in DnaK ${ }_{M m}$ was less complete than in $\mathrm{DnaK}_{E c}$. The other bands shown in the $1620-1500 \mathrm{~cm}^{-1}$ interval are due to amino acid side-chain absorption (Barth, 2000).

To obtain more information on $\mathrm{DnaK}_{\mathrm{Mm}}$ and $D_{\text {DnaK }}$ structures we compared the thermal denaturation patterns of these proteins. We collected infrared spectra as a function of temperature (in the range $20-95^{\circ} \mathrm{C}$ ). Figure $1 \mathrm{~B}$ shows as an example the infrared absorbance spectra of $\mathrm{DnaK}_{\mathrm{Mm}}$ collected at
20 and $90^{\circ} \mathrm{C}$. The amide $\mathrm{I}^{\prime}$ band intensity (absorbance) decreases with an increase in temperature, whereas the amide I'-band width (wavenumber) increases. An amide I' band shift also occurs. The

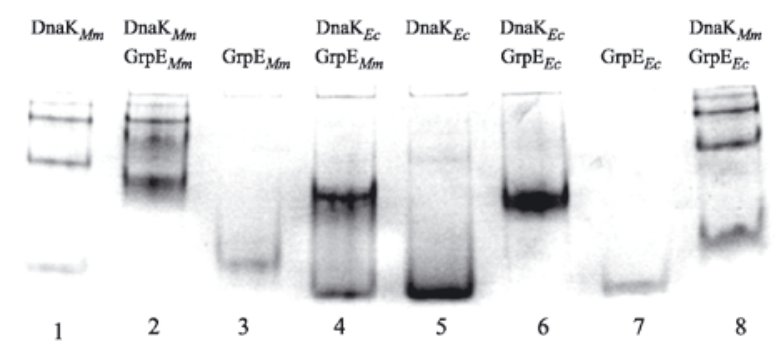

Figure 2. Formation of interspecies DnaK-GrpE complexes between the proteins from $E$. coli $\left(\mathrm{DnaK}_{E c}\right.$ and $\left.\operatorname{GrpE}_{E c}\right)$ and M. mazei $\left(\mathrm{GrpE}_{M m}\right.$ and $\left.\operatorname{DnaK}_{M m}\right)$.

The DnaK-GrpE complexes were studied by 10\% PAGE under non-denaturing conditions. Three micromoles of DnaK $_{M m}$ or DnaK $E_{E c}$ were incubated alone, or with $\mathrm{GrpE}_{M m}$ or $\operatorname{GrpE}_{E c}(3 \mu \mathrm{M}$ dimer $)$, in $50 \mathrm{mM}$ Tris $\mathrm{pH} 7.5,50 \mathrm{mM}$ $\mathrm{KCl}$ and $10 \mathrm{mM} \mathrm{MgCl}$ buffer, in combinations shown above the corresponding gel lanes, at $25^{\circ} \mathrm{C}$ for $15 \mathrm{~min}$. 
A

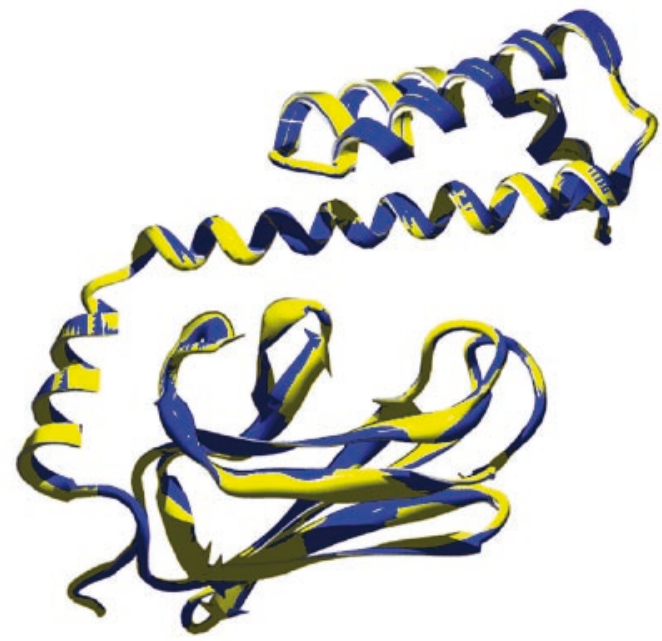

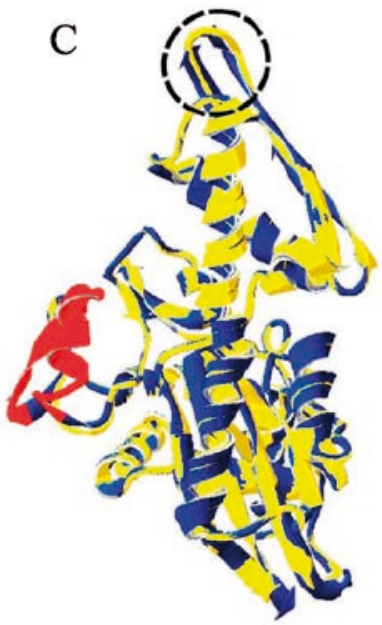

Figure 3. Comparison of structure of $\mathrm{DnaK}_{E c}$ to model of DnaK ${ }_{M m}$.

Models of the DnaK $\mathrm{K}_{\mathrm{Mm}}$ (yellow) ATPase and SBD domains were superimposed on the equivalent $D_{n a K}$ domain structure (violet). A. The SBDs of DnaK $\mathrm{K}_{M m}$ (yellow) and $\mathrm{DnaK}_{E c}$ (violet). The DnaK $E_{E c} S B D(1 D K X)$ was used as a template for modeling of the DnaK $\mathrm{K}_{M m}$ SBD. B and C. The predicted DnaK $\mathrm{K}_{M m}$ ATPase domain possesses a GrpE-binding loop like that in the DnaK $E_{E c}$. The ATPase domains of the archaeal and bacterial (1DKGD, used as a template for modeling of the archaeal domain) chaperones, in ribbon display, are shown viewed from the back (B) (as per Harrison et al., 1997), and from the side (C); the GrpE-binding loops are encircled. The 24-amino acid segment of DnaK ${ }_{E c^{\prime}}$ shown in red, is absent in DnaK $\mathrm{M}_{\mathrm{m}}$. thermal denaturation can be followed by measuring the amide $\mathrm{I}^{\prime}$ bandwidth at $3 / 4$ height, as marked in Fig. 1B. These parameters were used to calculate the thermal denaturation curves of the proteins, dis- played in Fig. 1C, as done previously (D'Auria et al., 2004). The curves were similar but $\mathrm{DnaK}_{M m}$ was more thermostable than was DnaK ${ }_{E c}$ : the $t_{\mathrm{m}}$ values of the proteins were $61.0^{\circ} \mathrm{C}$ and $56.4^{\circ} \mathrm{C}$, respectively
A

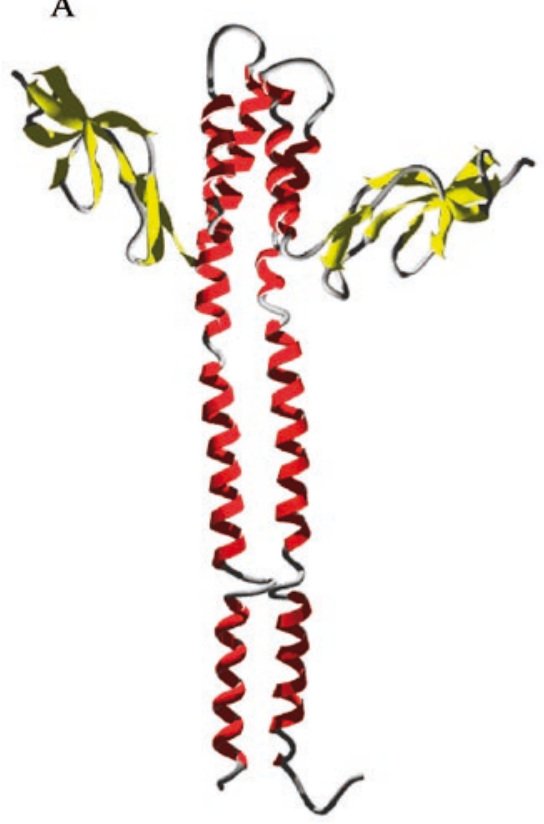

B

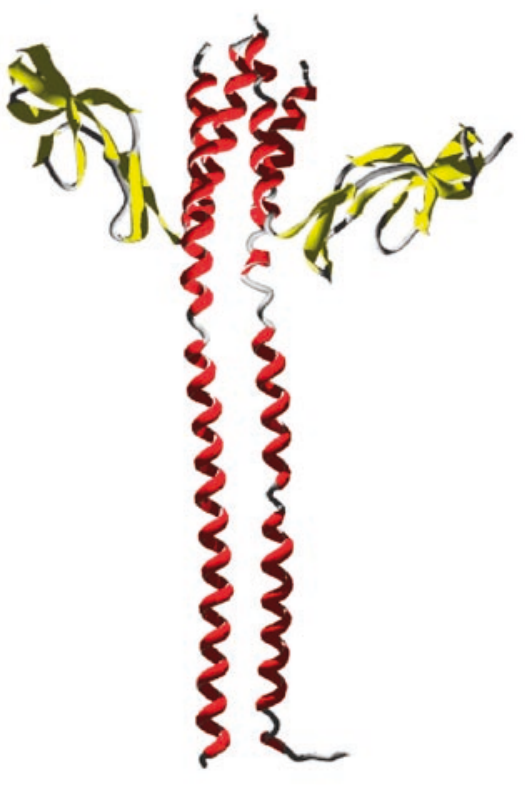

Figure 4. Comparison of structure of GrpE from E. coli to model of $\mathrm{GrpE}_{\mathrm{Mm}}$.

The $\mathrm{GrpE}_{E C}$ dimer structure (A). The model of $\mathrm{GrpE}_{M m}$ dimer constructed based on the solved structure of the E. coli DnaKGrpE complex (1DKG) (B). 
(Fig. 1C). Since the secondary structures of the two DnaKs analyzed were very similar, the higher $t_{\mathrm{m}}$ value of DnaK $\mathrm{Mm}_{m}$ may reflect the differences in tertiary or quaternary structure with respect to DnaK $E c$. The formation of intermolecular interactions (like during aggregation process) is usually accompanied by an increased amide I' bandwidth (D'Auria et al., 2004). Thus, the higher value of the amide I' bandwidth of $\mathrm{DnaK}_{M m}$ at $20^{\circ} \mathrm{C}$ as compared to $\mathrm{DnaK}_{E c}$ (Fig. 1C) may indicate that $\mathrm{DnaK}_{M m}$ forms oligomers. However, we must stress that the observed differences are small and the existence of the putative $\mathrm{DnaK}_{\mathrm{Mm}}$ oligomers requires further studies.

In conclusion, the FT-IR results showed a very high degree of similarity of the secondary structures of the DnaK proteins of M. mazei and E. coli, but with some small differences.

\section{Physical cooperation of DnaK and GrpE}

To assess the existence of interspecies "hybrid" DnaK-GrpE complexes, we incubated purified bacterial and archaeal DnaKs and GrpEs together, and then resolved the mixtures by native gel electrophoresis. Results presented in Fig. 2 showed that $D_{n a K}$ formed a complex with $\operatorname{GrpE}_{M m}$ (lane 4), and a similar complex was observed for DnaK $\mathrm{K}_{M m}$ and $\mathrm{GrpE}_{E c}$ (lane 8). Incidentally, the formation of the DnaK $E_{E c}-\mathrm{GrpE}_{M m}$ complex was used by us during purification of $\mathrm{GrpE}_{M m^{\prime}}$; the latter protein bound efficiently to $\mathrm{DnaK}_{E C^{-}}$ Sepharose affinity columns and, subsequently, the archaeal protein $\mathrm{GrpE}_{M m}$ was released in the presence of ATP (Zmijewski et al., 2004; and this work). DnaK ${ }_{M m}$ complexed more efficiently with $\operatorname{GrpE}_{M m}$ than with $\operatorname{GrpE}_{E c}$ (Fig. 2, lanes 2 and 8); this could contribute to the species specificity of DnaK $_{M m}$ observed in vivo (Zmijewski et al., 2004). DnaK $_{M m}$ migrated more slowly than the lowestmolecular-mass form of DnaK $E_{C c}$ (Fig. 2, lanes 1 and 5). It should be noted that DnaK $\mathrm{K}_{M m}$ tends to form highly oligomeric forms, which is visible in Fig. 2, lane 1. Since DnaK $\mathrm{K}_{\mathrm{Mm}}$ has a lower molecular mass than $\operatorname{DnaK}_{E c}\left(\right.$ DnaK $_{M m}=66288$ Da; DnaK $E c=69076$ $\mathrm{Da})$, the slower migration of the lowest molecular form of DnaK $\mathrm{Mm}_{m}$ is probably due, at least partially, to differences in the shape of the molecules, since the calculated isoelectric points are quite similar (pI of $D_{n a K}$ and $D_{n a K}$ is 4.89 and 4.83, respectively).

\section{DISCUSSION}

It is currently believed that the archaeal Hsp70 system arose by lateral transfer of the chaperone genes from bacteria. This hypothesis is based on the sequence analysis of the known Hsp70 genes (Gupta \& Singh, 1992; Gribaldo et al., 1999; Macario \& Conway De Macario, 1999; Macario et al., 2004). Our previous finding that the DnaK and GrpE proteins of $M$. mazei can functionally cooperate with the E. coli GrpE and DnaK proteins supported this hypothesis (Zmijewski et al., 2004). The aim of this work was to investigate the molecular basis of the observed interspecies chaperone-co-chaperone interaction.

We have shown, using FT-IR spectroscopy, a high similarity of the secondary structures of the archaeal and bacterial DnaK proteins (Fig. 1A). This forms a good foundation for the observed DnaKGrpE interspecies cooperation.

To better understand the molecular background of this interaction, we modeled the ATPase (Fig. 3B and C) and SBD (Fig. 3A) domains of $\mathrm{DnaK}_{\mathrm{Mm}}$, basing on the solved crystal structures of the respective DnaK $E_{E C}$ domains. We found that superimposition of the ATPase domains from $\mathrm{DnaK}_{M m}$ and $D_{n a K}$ gave a calculated root-mean-square deviation (rmsd) for 347 equivalent backbone atoms $(\mathrm{N}, \mathrm{C} \alpha, \mathrm{C})$ of the ATPase domain of $\mathrm{DnaK}_{\mathrm{Mm}}$ of 0.26 $\AA$ (0.22 for $C \alpha)$. Superimposition of the modeled $D_{n a K}$ SBD and the solved structure of the DnaK ${ }_{E c}$ SBD gave a calculated rmsd of $0.1 \AA$ for 205 equivalent backbone atoms $(\mathrm{N}, \mathrm{C} \alpha, \mathrm{C})$, and 0.09 for the $\mathrm{C} \alpha$ atoms of DnaK $\mathrm{M}_{m}$ SBD. These values indicate a high degree of similarity between $\mathrm{DnaK}_{M m}$ and $\mathrm{DnaK}_{E c}$ both in the ATPase domain (Fig. 3B and C) as well as in the SBD (Fig. 3A).

Since the functional cooperation of DnaK and GrpE requires a physical interaction of DnaK with a GrpE dimer (Schonfeld et al., 1995; Harrison et al., 1997; Harrison, 2003), we have analyzed the formation of the DnaK-GrpE complexes by electrophoresis under non-denaturing conditions. This experiment revealed that indeed the hybrid $\operatorname{DnaK}_{M m}-\mathrm{GrpE}_{E c}$ and $D_{n a K}-G_{E c} E_{M m}$ complexes were formed efficiently (Fig. 2). Interaction of $\mathrm{DnaK}_{E c}$ with $\mathrm{GrpE}_{E c}$ involves several contact regions in both molecules, as seen from the solved structure of the $\mathrm{GrpE}_{E C}$ dimer bound to the ATPase domain of $\operatorname{DnaK}_{E c}$ (Harrison et al., 1997). DnaK ${ }_{M m^{\prime}}$ like bacterial DnaK but unlike eukaryotic Hsp70, possesses in its ATPase domain a conserved loop (circled in Fig. 3B and C), which in bacteria plays a key role in GrpE binding (Buchberger et al., 1994). Other sites involved in DnaK-GrpE interactions are also conserved between the two molecules; for example, the loop formed by amino acids 43 to 47 , and the amino acid glycine 32 (not shown), are present both in $\mathrm{DnaK}_{E c}$ and $\mathrm{DnaK}_{M m}$. Furthermore, the model of $\operatorname{GrpE}_{M m}$ (Fig.4B), based on the solved structure of the $\mathrm{GrpE}_{E c}$ dimer (Fig. $4 \mathrm{~A}$ ), predicted that the structure of $\mathrm{GrpE}_{\mathrm{Mm}}$ was very similar, lending support to the observed fact 
that the archaeal and the bacterial molecules can interact and assemble into a functional chaperone machine, even though the two GrpEs have relatively few amino acids in common. It is worth noting here that the mitochondrial and bacterial GrpEs, in spite of low sequence homology, can be exchanged (Choglay et al., 2001). A comparison of the sequence homology showed that the M. mazei GrpE, like that of the E. coli GrpE, is more similar to isoform 2 than to isoform 1 of the mammalian mitochondrial GrpE.

Apart from the discussed similarity of the $\mathrm{DnaK}_{M m}$ and $\mathrm{DnaK}_{E c}$ structures, we have found some differences. As predicted by sequence data and modeling (Fig. 3B and C), the ATPase domains differ in one significant structural feature. In the DnaK $_{E c}$ ATPase domain, there is a 24-amino-acid segment (positions 75-98) that forms a loop consisting of two $\beta$-strands separated by an $\alpha$-helix (Fig. 3B and $C$ ). This segment is present in the DnaKs from Gram-negative bacteria and in the Hsp70 of eukaryotes, but it is missing in the DnaKs from Grampositive bacteria and archaea (Macario et al., 1991; Gupta \& Singh, 1992). The physiological role of this region is unknown and, to our knowledge, there are no genetic or biochemical data indicating that it is involved in the GrpE binding.

There were some small differences shown by FT-IR spectroscopy. (1) In the $\mathrm{DnaK}_{M m}$ spectrum, one component (representing $\beta$-structures) was lacking at $1628 \mathrm{~cm}^{-1}$, which was present in $\mathrm{DnaK}_{E c}$ (Fig. 1A). This difference may be due to the absence, in the GrpE-binding loop of the DnaK ${ }_{M m}$ ATP-binding domain, of the $\beta$-structure that is present at this location in $D_{n a K}$ (Fig. 3B and C). This putative structural difference might explain why $\mathrm{DnaK}_{M m}$ complexed more efficiently with $\mathrm{GrpE}_{M m}$ than with $\operatorname{GrpE}_{E c}$ (Fig. 2). (2) The band at $1652.8 \mathrm{~cm}^{-1}$, representing $\alpha$-helices, was shifted towards the higher wavenumbers in the DnaK $\mathrm{Mm}_{\mathrm{m}}$ spectrum (Fig. 1A), indicating that these structures are less exposed to the solvent in $\mathrm{DnaK}_{M m}$ than in DnaK ${ }_{E c}$. Modeling of the ATPase domain revealed that the 24-amino-acid segment absent in the archaeal protein corresponds to an $\alpha$-helix that is exposed to the surface (Fig. 3B and C). The lack of this 24-amino-acid segment may have contributed to the observed band shift. (3) The band at $1636 \mathrm{~cm}^{-1}$, representing $\beta$-structures, was shifted towards the lower wavenumbers in the $\mathrm{DnaK}_{M m}$ spectrum; these $\beta$-structures are more exposed to the solvent in $\mathrm{DnaK}_{M m}$ than in DnaK ${ }_{E c}$. This difference between the spectra could be due to the exposure of the $\beta$-sheets in the ATPase domain of $\mathrm{DnaK}_{\mathrm{Mm}}$ caused by the absence of the 24-amino-acid $\alpha$-helix discussed above. It is also possible that the SBD, composed mainly of $\beta$-sheets, is more widely open in $\mathrm{DnaK}_{M m}$ than in $\mathrm{DnaK}_{E c}$. (4) The band at 1547.2 $\mathrm{cm}^{-1}$, representing the residual amide II band, was higher in the spectrum of DnaK $\mathrm{Mm}_{\mathrm{Mm}}$ than in the spectrum of $\operatorname{DnaK}_{E c}$ (Fig. 1A), indicating less deuterium exchange for the archaeal protein. DnaK $\mathrm{Mm}_{m}$ appears to be generally more compact and/or less flexible than $D_{n a K_{E c^{\prime}}}$ a possibility supported by the results of thermal-stability measurements that showed a higher temperature stability for $\mathrm{DnaK}_{M m}$ (Fig. 1C). It is possible that the higher thermostability of $\mathrm{DnaK}_{\mathrm{Mm}}$ might be caused by the fact that $\mathrm{DnaK}_{\mathrm{Mm}}$ tends to form oligomers (Fig. 2, and results not shown).

The above-discussed small differences between DnaK $\mathrm{K}_{M m}$ and $\mathrm{DnaK}_{E C^{\prime}}$ shown by FT-IR, do not preclude GrpE $E_{E c}$ binding by DnaK ${ }_{M m^{\prime}}$ however, they may be one of the reasons why E. coli dnaK mutants are not complemented by the M. mazei dnaK gene (Zmijewski et al., 2004).

In conclusion, the experimental data supported by modeling showed a high similarity of the secondary structures of $\mathrm{DnaK}_{M m}$ and $\mathrm{DnaK}_{E c}$. In addition, modeling suggested a similarity of the 3dimensional structures of these chaperones, and of the archaeal and bacterial GrpE proteins. We believe that these similarities form the structural basis for the formation of the DnaK-GrpE interspecies hybrid complexes. Our results, showing an overall structural similarity of the bacterial and archaeal DnaK proteins and suggesting such similarity of the GrpE proteins are a further support for the theory of lateral gene transfer from bacteria to archaea.

\section{Acknowledgements}

We would like to express our gratitude to Everly Conway de Macario for excellent advice and for critical readings of the manuscript and the Photography and Illustration Unit of the Wadsworth Center for help with figures.

This work was supported by a grant from the State Committee for Scientific Research (KBN, Poland, 374/P04/99/16) and by a grant from Università Politecnica delle Marche (ricerche di Ateneo). The initial part of this work contributed by A.J.L.M. was done at the Wadsworth Center, New York State Department of Health, Albany, NY, USA with partial support from the San Francisco Foundation.

\section{REFERENCES}

Arrondo JL, Muga A, Castresana J, Goni FM (1993) Quantitative studies of the structure of proteins in solution by Fourier-transform infrared spectroscopy. Prog Biophys Mol Biol 59: 23-56.

Barth A (2000) The infrared absorption of amino acid side chains. Prog Biophys Mol Biol 74: 141-173.

Bradford MM (1976) A rapid and sensitive method for the quantitation of microgram quantities of protein utilizing the principle of protein-dye binding. Anal Biochem 72: $248-254$. 
Buchberger A, Schroder H, Buttner M, Valencia A, Bukau B (1994) A conserved loop in the ATPase domain of the DnaK chaperone is essential for stable binding of GrpE. Nat Struct Biol 1: 95-101.

Bukau B, Deuerling E, Pfund C, Craig EA (2000) Getting newly synthesized proteins into shape. Cell 101: 119122.

Bukau B, Weissman J, Horwich A (2006) Molecular chaperones and protein quality control. Cell 125: 443-451.

Byler DM, Susi H (1986) Examination of the secondary structure of proteins by deconvolved FTIR spectra. Biopolymers 25: 469-487.

Choglay AA, Chapple JP, Blatch GL, Cheetham ME (2001) Identification and characterization of a human mitochondrial homologue of the bacterial co-chaperone GrpE. Gene 267: 125-134.

D'Auria S, Alfieri F, Staiano, M, Pelella, F, Rossi, M, Scire, A, Tanfani, F, Bertoli, E, Grycznyski, Z, Lakowicz, JR (2004) Structural and thermal stability characterization of Escherichia coli $\mathrm{D}$-galactose/D-glucose-binding protein. Biotechnol Prog 20: 330-337.

Deuerling E, Bukau B (2004) Chaperone-assisted folding of newly synthesized proteins in the cytosol. Crit Rev Biochem Mol Biol 39: 261-277.

Erbse A, Mayer MP, Bukau B (2004) Mechanism of substrate recognition by Hsp70 chaperones. Biochem Soc Trans 32: 617-621.

Gribaldo S, Lumia V, Creti R, de Macario EC, Sanangelantoni A, Cammarano P (1999) Discontinuous occurrence of the hsp70 (dnaK) gene among Archaea and sequence features of HSP70 suggest a novel outlook on phylogenies inferred from this protein. J Bacteriol 181: 434-443.

Guex N, Peitsch MC (1997) SWISS-MODEL and the SwissPdbViewer: an environment for comparative protein modeling. Electrophoresis 18: 2714-2723.

Gupta RS, Singh B (1992) Cloning of the HSP70 gene from Halobacterium marismortui: relatedness of archaebacterial HSP70 to its eubacterial homologs and a model for the evolution of the HSP70 gene. J Bacteriol 174: 4594-4605.

Harrison C (2003) GrpE, a nucleotide exchange factor for DnaK. Cell Stress Chaperones 8: 218-224.

Harrison CJ, Hayer-Hartl M, Di Liberto M, Hartl F, Kuriyan J (1997) Crystal structure of the nucleotide exchange factor GrpE bound to the ATPase domain of the molecular chaperone DnaK. Science 276: 431-435.

Jackson M, Mantsch HH (1992) Halogenated alcohols as solvents for proteins: FTIR spectroscopic studies. Biochim Biophys Acta 1118: 139-143.

Krimm S, Bandekar J (1986) Vibrational spectroscopy and conformation of peptides, polypeptides, and proteins. Adv Protein Chem 38: 181-364.

Kultz D (2005) Molecular and evolutionary basis of the cellular stress response. Annu Rev Physiol 67: 225-257.

Laemmli UK (1970) Cleavage of structural proteins during assembly of the head of bacteriophage T4. Nature 227: 680-685.

Macario AJ, Conway De Macario E (1999) The archaeal molecular chaperone machine: peculiarities and paradoxes. Genetics 152: 1277-1283.
Macario AJ, Conway De Macario E (2001) The molecular chaperone system and other anti-stress mechanisms in archaea. Front Biosci 6: D262-283.

Macario AJ, Dugan CB, Conway de Macario E (1991) A dnaK homolog in the archaebacterium Methanosarcina mazei S6. Gene 108: 133-137.

Macario AJ, Malz M, Conway de Macario E (2004) Evolution of assisted protein folding: the distribution of the main chaperoning systems within the phylogenetic domain archaea. Front Biosci 9: 1318-1332.

Mayer MP, Schroder H, Rudiger S, Paal K, Laufen T, Bukau B (2000) Multistep mechanism of substrate binding determines chaperone activity of Hsp70. Nat Struct Biol 7: 586-593.

Meersman F, Smeller L, Heremans K (2002) Comparative Fourier transform infrared spectroscopy study of cold-, pressure-, and heat-induced unfolding and aggregation of myoglobin. Biophys J 82: 2635-2644.

Pedone E, Bartolucci S, Rossi M, Pierfederici FM, Scirè A, Cacciamani T, Tanfani F (2003) Structural and thermal stability analysis of Escherichia coli and Alicyclobacillus acidocaldarius thioredoxin revealed a molten globulelike state in thermal denaturation pathway of the proteins: an infrared spectroscopic study. Biochem J 373: 875-883.

Peitsch MC (1996) ProMod and Swiss-Model: Internetbased tools for automated comparative protein modelling. Biochem Soc Trans 24: 274-279.

Salooma P, Schaleger LL, Long FA (1964) Solvent deuterium isotope effects on acid-base equilibria. J Am Chem Soc 86: $1-7$.

Schonfeld HJ, Schmidt D, Schroder H, Bukau B (1995) The DnaK chaperone system of Escherichia coli: quaternary structures and interactions of the DnaK and GrpE components. J Biol Chem 270: 2183-2189.

Tanfani F, Galeazzi T, Curatola G, Bertoli E, Ferretti G (1997) Reduced $\beta$-strand content in apoprotein B-100 in smaller and denser low-density lipoprotein subclasses as probed by Fourier-transform infrared spectroscopy. Biochem J 322 (Pt 3): 765-769.

Truscott KN, Brandner K, Pfanner N (2003) Mechanisms of protein import into mitochondria. Curr Biol 13: R326337.

Young JC, Agashe VR, Siegers K, Hartl FU (2004) Pathways of chaperone-mediated protein folding in the cytosol. Nat Rev Mol Cell Biol 5: 781-791.

Zhu X, Zhao X, Burkholder WF, Gragerov A, Ogata CM, Gottesman ME, Hendrickson WA (1996) Structural analysis of substrate binding by the molecular chaperone DnaK. Science 272: 1606-1614.

Zmijewski MA, Macario AJ, Lipinska B (2004) Functional similarities and differences of an archaeal Hsp70(DnaK) stress protein compared with its homologue from the bacterium Escherichia coli. J Mol Biol 336: 539-549. 